\title{
A Decision-Support Tool to Deal with the Strategies Alignment Process in Collaborative Networks
}

\author{
Beatriz Andres ${ }^{1}$, Raul Poler ${ }^{1}$, Joao Rosas ${ }^{2,3}$, Luis Camarinha-Matos ${ }^{2,3}$ \\ ${ }^{1}$ Research Centre on Production Management and Engineering (CIGIP). Universitat \\ Politècnica de València (UPV). Calle Alarcón, 03801 Alcoy, Spain \\ ${ }^{2}$ Faculty of Sciences and Technology, New University of Lisbon, Portugal \\ ${ }^{3}$ Uninova Institute, Centre of Technology and Systems, Portugal \\ \{bandres, rpoler\}@ cigip.upv.es, \{jrosas, cam\}@ uninova.pt
}

\begin{abstract}
The alignment of strategies among the enterprises that belong to collaborative networks is of increasing importance due to the influence on the networks operation success in the long term. This paper proposes a DecisionSupport Tool for Strategies Alignment (DST-SA) to support SMEs in the selection of strategies that allow higher levels of alignment amongst all the strategies formulated by each partner. The DST-SA includes a mathematical model, a simulation software, and a programmed application, to address the strategies alignment process from a collaborative perspective. The result of the DST-SA is the identification of the strategies that are aligned and the proper time to activate these strategies with the main aim of obtaining higher levels of network performance.
\end{abstract}

Keywords: collaborative networks; strategies alignment; decision-support tool

\section{Formulation of the Research Question and Its Motivation}

Small and Medium Enterprises (SMEs) are currently more conscious about the benefits of participating in Collaborative Networks (CN), due to higher levels of competitiveness, agility, responsiveness, and adaptability that are acquired through collaboration. These characteristics allow them to rapidly face the market evolutions and the dynamicity induced by the globalization process. On the other hand, the participation of SMEs in $\mathrm{CN}$ has a number of associated challenges, which derive from the lack of resources and capabilities, and the limitations associated to cultural barriers. Generally speaking, European SMEs do not currently have access to advanced collaborative decision-support tools due to their limited resources.

In general, a $\mathrm{CN}$ consists of heterogeneous and autonomous partners [1], each one defining its own objectives and formulating its own business strategies. In this context, a wide variety of strategies can be formulated by each of the enterprises that belong to the $\mathrm{CN}$, with the aim of reaching their defined objectives. Therefore, contradictions between the strategies formulated by one enterprise and the objectives defined by another enterprise of the network could appear, resulting in potential conflicts and selfish behaviours. Lets describe an intuitive example considering two 
enterprises that acquire the role of manufacturer and distributor. Each one defines two objectives and two strategies. The manufacturer, $O b j_{1 M n f}$. Reduce the production cost by $10 \%, \operatorname{Obj}_{2 M n f}$ : Reduce fluctuations in production, $\operatorname{St}_{I M n f}$ : Use lower quality packaging, Str ${ }_{2 M n f}$ : Establish a collaborative production planning; and the Distributor: Obj $j_{1 \text { Dis: }}$ Increase the net demand by $10 \%$ in an exclusive market, Obj $j_{2 D i s}$ : Sell all the stock next to expire, Str ${ }_{1 D i s}$ : Promote the image of an exclusive product, $\operatorname{Str}_{2 \text { Dis: }}$ : Acquire a decision support system in the forecast demand process. Using a logical reasoning, a misalignment is observed when activating $\operatorname{Str}_{1 M n f}$ due to it has negative influences with the Obj ${ }_{1 D i s}$, whilst $\operatorname{Str}_{2 M n f}$ positively influences all the objectives defined by the distributor. This is an intuitive example but when a large amount of enterprises face the decision-making of selecting business strategies the problem increases in difficulty to be solved, specially when considering the $\mathrm{CN}$ context.

It is therefore important to study the strategies alignment process, and provide collaborative enterprises with support to proper selection of strategies in order to avoid the activation of misaligned strategies among the $\mathrm{CN}$ partners. The main aim of such process is to reduce the lack of coherence and concordance in the pool of strategies activated by each of the enterprises belonging to the $\mathrm{CN}$. The activation of a proper combination of well-aligned strategies reduces the emergence of conflicts within the $\mathrm{CN}$, ensuring its sustainability and convenient operation in the long term.

Despite the importance of the concept of alignment in the $\mathrm{CN}$ operation, there is a gap in the literature of adequate methods to formally represent and solve the strategies alignment process. More specifically, there is a lack of approaches that consider (i) the whole set of strategies formulated by the network partners, and (ii) the influence that each of these strategies exert on the wide diversity of objectives defined by each enterprise. Besides this, and to the best of our knowledge, it does not exist any decision support tool that guides the selection of strategies based on the alignment characteristic. Motivated by this situation, the following research questions are addressed:

1. What would be an adequate decision support tool to guide enterprises in the selection of strategies?

2. Amongst all strategies formulated by each enterprise belonging to a $\mathrm{CN}$, which strategies should be selected for achieving high levels of alignment?

The strategies alignment process involves modelling the impacts that such strategies, once activated, have on the objectives [2]. The heterogeneity and autonomy that characterises the $\mathrm{CN}$ enterprises and the multiplicity of the information exchanges related with the formulated strategies and the defined objectives, requires new approaches to cope with the complexity of the process. Consequently, SMEs need a decision support tool to help them achieving a global view of the strategies and objectives with the aim to respond faster and more efficiently to potential contradictions and misalignments. As such, a Decision-Support Tool is proposed to deal with the Strategies Alignment process in CN (DST-SA). DST-SA provides an affordable tool to SMEs with the aim of helping them to reach high levels of alignment among the activated strategies, thus contributing to increase their competitiveness in the global economy.

The remaining of this paper includes identifying in which ways the research in business strategies alignment can benefit from cyber-physical systems (Section 2). In Section 3, a brief state of the art on the strategies alignment process is provided. The 
A Decision-Support Tool to Deal with the Strategies Alignment Process 5

research contribution and innovation, regarding the proposed DST-SA tool, is described in Section 4. Finally, in Section 5 the conclusions of the work, some discussion, and further research lines are introduced.

\section{Cyber Physical Systems}

The integration of computing, communication, and control technologies has led to developments in intelligent sensors and a great level of integration between the software and the physical world [3]. The term Cyber Physical Systems (CPS) was devised by Lee [4] motivated by the relevance of the interactions between computation and physical worlds, encouraging real-time information extraction, transmission and analysis for intelligent monitoring, decision-making and control [5]. In this context, CPS, and more specifically the Internet of Things (IoT), are opening a new dimension for innovation, higher levels of integration in enterprise networks, and consequently challenging their business strategies. With CPS, enterprises have much wider and fast access to real-world information, contributing to the notion of "sensing enterprise". This allows enterprises to anticipate future decisions by using multidimensional data, captured through physical and virtual objects, and generating added value information [6]. Enriching enterprises' awareness through intelligent, interconnected, and interoperable smart components and devices, empowers enterprises systems, making them responsive in real time to events related with business strategies.

In the context of decision making for the selection of aligned business strategies, the access to relevant information, among the collaborative partners, is a decisive issue. The use of devices embedded in the enterprises' business environment provides access to larger amounts of information in the decision-making process. Furthermore, access to real time information allows enterprises to be more agile when they need to make adjustments in business strategies, in case a misalignment appears.

The consideration of CPS requires new approaches to strategies alignment. On one hand they need to be developed taking into account this "new referential"; and on the other hand additional requirements and complexity related with the decision making for the selection of strategies, considering a collaborative perspective, increase the need to make the right decisions (high quality decisions) for the whole of the $\mathrm{CN}$ partners. As such, there is a need for (i) proper modelling of the relevant variables contained in the strategies alignment process, as well as their interdependencies and influences, and (ii) a decision support tool to help in identifying and assessing strategies alignment process.

\section{State of the Art}

Different models, guidelines, methods, and tools have been proposed in the literature to address the business strategies alignment process. Nevertheless, the conducted analysis revealed that the contributions, provided so far, refer to the alignment of particular network strategies, such as supply strategies [7][8], marketing strategies [9], 
or product design strategies [10]. Despite the importance of the alignment process, namely in terms of avoiding partnership conflicts, to the best of our knowledge, there is a lack of approaches modelling the alignment of the different strategies formulated by individual and heterogeneous enterprises belonging to the $\mathrm{CN}$. Therefore, a holistic approach that allows modelling the influences that all strategies formulated by all partners have on the wide diversity of defined objectives is needed. In order to fill this gap, an integrated approach to holistically model the strategies alignment process has been previously proposed [2] [11] [12]. The strategies alignment model (SAM) [11] deals with the strategies and objectives regardless of their nature and type, considering a $\mathrm{CN}$ context. Taking this baseline, a novel decision-support tool is proposed in this paper, which aims at supporting enterprises in the automatic construction of the SAM and allow SMEs to succeed in the establishment of sustainable long-term collaborative relationships using the business strategies alignment mechanism.

\section{Research Contribution and Innovation in DST-SA}

The research contribution of this paper extends previous work of Andres and Poler [2] [11] [12] [13] in which a model to represent the influences between the defined business objectives and the strategies formulated by each of the networked partners, as well as an associated method, the System Dynamics (SD) [14], are proposed to solve the strategies alignment process. The main aim is to identify the set of strategies to activate, while guaranteeing their alignment. Hereafter, and in order to facilitate the understanding of DST-SA, these works are briefly described. The Strategies Alignment Model (SAM) proposes a mathematical representation of the influences and relations between either the improvement or worsening of KPIs when a set of strategies are activated. Potential misalignments among the strategies are identified by the SAM implementation. Thus, its application leads to obtain the set of aligned strategies that positively influence the majority of the objectives defined by the networked partners. The optimization function of SAM focuses on the network level, so that the identified strategies maximise the positive influences (or minimize the negative influences) on the defined KPIs. The decision variables used in SAM for the maximisation function are (i) the number of units of the strategy $\left(u_{-} s t r_{i s}\right)$ to be activated, and (ii) the time when the strategies have to be activated $\left(t i \_s t r_{i s}\right)$. The SD [14] is the method selected to solve the SAM, as it allows representing the causal influences between the strategies and the objectives achievement (through KPIs), within the complex system formed by the enterprises of a CN. The SD method classifies the parameters of SAM using the stock variables, flow variables, and auxiliary variables [12].

The Decision-Making Tool for the Strategies Alignment (DST-SA) is proposed for solving SAM through a SD simulation approach. The DST-SA is based on a performance measurement schema that allows estimating, from a quantitative perspective, the value influences that an activated strategy has on the KPIs used to measure each objective defined by each $\mathrm{CN}$ enterprise. A simulation tool is thus included in DST-SA to automatically solve the proposed model, assessing and 
supporting the strategies alignment process. AnyLogic simulation software [15] was selected as a support component of DST-SA, since it allows representing, in SD, the elements of SAM. The optimisation package included in the simulation software allows to automatically solve SAM and obtain the solution of the decision variables $\left(u \_s t r_{i s}, t i \_s t r_{i s}\right)$ that maximize the network's performance [12]. Moreover, DST-SA includes a component to automatically generate the model introduced in [2], [13] for the strategies alignment process under a SD approach.

The manual modelling of the SAM [2] [11], under SD simulation [12] could be feasible with a small number of enterprises. However, when the modeller faces a network with a high number of enterprises, each one defining several KPIs and formulating a high number of strategies, the amount of parameters, auxiliary variables, flow variables, and stock variables defined in SAM tend to increase exponentially. This results in the increase of the size of the problem to be modelled, which becomes difficult to handle manually. In order to avoid such tedious task, DSTSA includes a component that automatically generates the SAM for the simulation software: the Strategies Alignment model GENerator (SAGEN). DST-SA also includes a database with all data required for building the SAM in SAGEN and feed the SAM to the SD simulation software.

For the design of SAGEN component, the authors assume that models built in AnyLogic SD simulation software have the property of being read in XML language (Extensible Markup Language) with a specific schema. SAGEN allows building an XML file, containing the structured schema of SAM in SD notation, representing the enterprises, objectives, strategies and the influences between them. In order to reproduce the SAM specific schema in SD, the structure of an XML file created by AnyLogic is analysed. An example of the XML notation used to represent the Flow and Stock variables in SAM is depicted in Table 1.

Table 1. XML Schemas to define Flow and Stock Variables
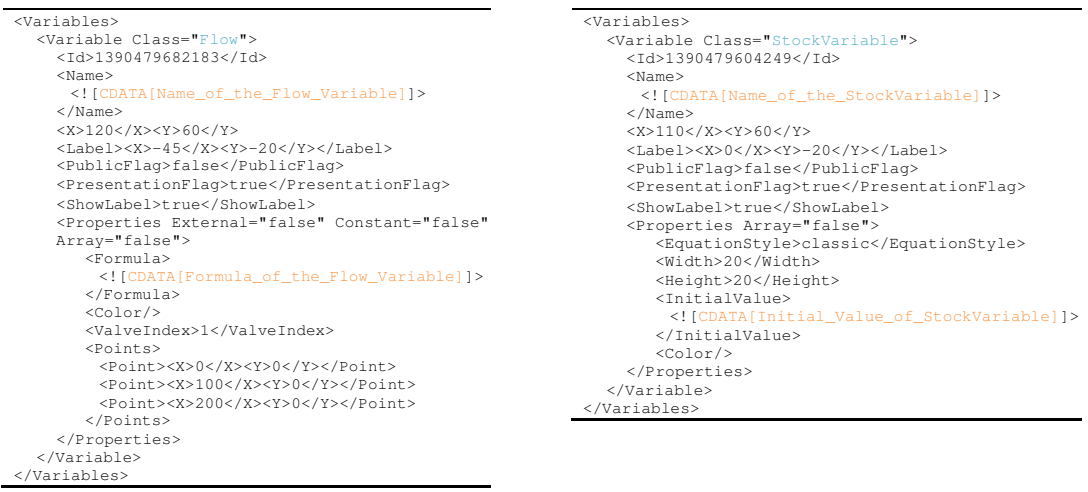

From a technical point of view, Lazarus [16] has been used as an Integrated Development Environment (IDE) for the SAGEN development. Lazarus is an open source and free alternative to Delphi, developed as open source project from Free Pascal. Therefore, Pascal programing language was used to build SAGEN, due to its simplicity and easiness to expand the SAGEN component for its use in other domains. 


\section{B. Andres et al.}

The obtained XML file contains all variables and data required to feed the SAM, which is readable by AnyLogic simulation software (see Figure 1). Besides generating XML code, SAGEN offers a user-friendly interface that allows the enterprises to enter the data required to feed the SAM (Figure 2). The procedure followed in SAGEN is: (i) introduce all the required data to feed the SAM through the interface, (ii) the data is stored in a Microsoft Access Database using a OCDBConnection, (iii) the procedures described in Lazarus IDE allow to create XML file with all the elements to build both the flow diagram of the SAM (simulation experiment) in SD, and the optimization experiment to be opened in the AnyLogic simulation software. SAGEN creates a structured positioning of all objects that form the SAM, increasing the readability and understanding of the model. Figure 3 includes an example of two enterprises, each one formulating two strategies and defining two KPIs.

Summarising, the proposed DST-SA consists of the SAM, the SD-based simulation software, and the SAGEN component to automatically generate the SAM in a format accepted by the Anylogic simulation software. With DST-SA, enterprises will be able to build, solve, and assess the strategies alignment process from a collaborative perspective. The network enterprises collaboratively make the decision of identifying the aligned strategies to be activated, and the time frame when to activate them so that the performance of the network is maximised.

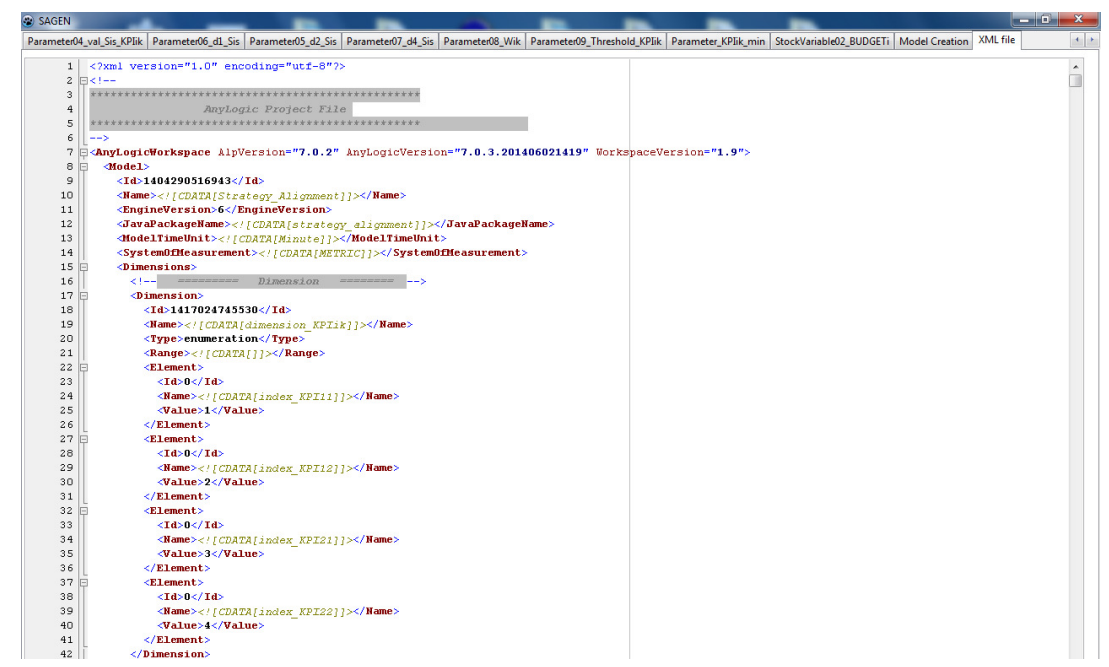

Fig. 1. XML file: strategies alignment simulation model in XML language

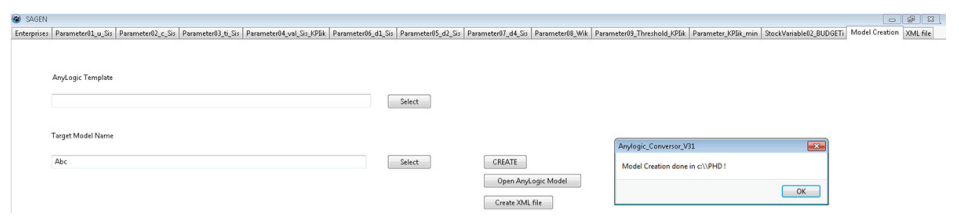

Fig. 2. SAGEN component interface 


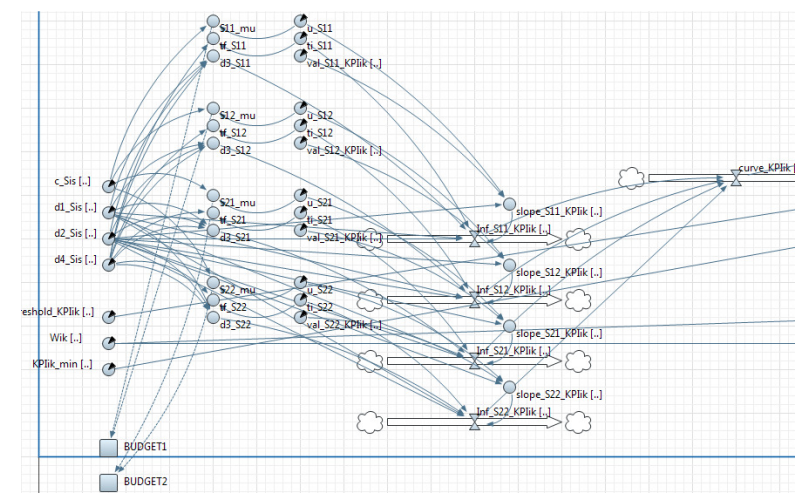

Fig. 3. SAM simulation experiment built with SAGEN

The DST-SA supports the decision-making regarding the selection of those strategies that exert positive influences on the majority of the defined objectives (being the negative influences minimized), and deals with misalignments, reducing potential conflicts.

\section{Conclusions and Further Work}

In this paper we have presented a decision support tool called DST-SA to help in the selection of aligned business strategies in a CN context. The tool rests on three pillars: the strategies alignment model (SAM) based on SD, the simulation software (AnyLogic), and application component (SAGEN) that automatically generates the SD model for the simulation software. DST-SA allows considering from a holistic perspective all the strategies formulated by each of the network partners and the influences that their activation would have on each of the performance objectives defined at the enterprises level. The SAGEN module significantly facilitates the collaborative process of strategies alignment, so that the construction of the model in the SD simulation software is automated. The SAM embedded in the DST-SA aims at maximising the network performance. Thus, not all $\mathrm{CN}$ partners necessarily experience an improvement in their performance level, being possible that part of the partners improve their performance at the expense of a loss of other partners. However, in the global context of the $\mathrm{CN}$, the performance will be improved, contributing to its sustainability, and thus, also improving the long-term relationships among the collaborative partners.

The utilization of the DST-SA will serve (i) to show SMEs how they are currently making decisions when selecting the strategies to activate, (ii) to show SMEs how to collaboratively make decisions with the main aim of selecting aligned strategies, (iii) to train companies in the decision-making process so that they collaboratively perform the selection of aligned strategies. DST-SA allows decision makers to get a new vision of the problem of selecting strategies, from a global perspective within the $\mathrm{CN}$. Hence, decision-makers do not only consider the achievement of the objectives 
of their company, but also take into account the influences that strategies have on the objectives of other network partners. Future research includes the assessment of DST$\mathrm{SA}$ in real CNs, considering various network sizes and industrial sectors and performing a sensitivity analysis to estimate the robustness of the tool to perturbations in objectives and strategies. The development of a methodology to guide SMEs in the strategies alignment process must be performed; for assessing of the strategies selection considering decentralised scenarios, and fostering negotiation processes.

Acknowledgments. This work was supported in part by Programa Val $i+d$ para investigadores en formación (ACIF) and Uninova-CTS through FCT (Fundação para a Ciência e Tecnologia) - PEST program UID/EEA/00066/2013 (Impactor project).

\section{References}

1. L. M. Camarinha-Matos and H. Afsarmanesh, "Collaborative networks : a new scientific discipline," J. Intell. Manuf., vol. 16, no. 4-5, pp. 439-452, 2005.

2. B. Andres and R. Poler, "Dealing with the Alignment of Strategies within the Collaborative Networked Partners," IFIP Int. Fed. Inf. Process., vol. 450, pp. 13-21, 2015.

3. X. Yue, H. Cai, H. Yan, C. Zou, and K. Zhou, "Microprocessors and Microsystems Cloudassisted industrial cyber-physical systems : An insight," Microprocess. Microsyst., 2015.

4. E. A. Lee, "Cyber-physical systems-are computing foundations ade- quate?," in NSF Workshop on Cyber-Physical Systems: Research Motivation, Techniques and Roadmap, 2006, pp. 1-9.

5. X. Yue, H. Cai, H. Yan, C. Zou, and K. Zhou, "Microprocessors and Microsystems Cloudassisted industrial cyber-physical systems : An insight," Microprocess. Microsyst., 2015.

6. L. M. Camarinha-Matos, J. Goes, L. Gomes, and J. Martins, "Contributing to the Internet of Things," IFIP Adv. Inf. Commun. Technol., vol. 394, no. April, pp. 3-12, 2013.

7. P. D. Cousins, "The alignment of appropriate firm and supply strategies for competitive advantage," Int. J. Oper. Prod. Manag., vol. 25, no. 5, pp. 403-428, May 2005.

8. J. Ashayeri and W. Selen, "Global sourcing strategy alignment using business intelligence : a conceptual framework," Int. J. Procure. Manag., vol. 1, no. 3, pp. 342 - 358, 2008.

9. K. W. Green, D. Whitten, and R. A. Inman, "Aligning marketing strategies throughout the supply chain to enhance performance," Ind. Mark. Manag., vol. 41, no. 6, pp. 1008-1018, 2012.

10.C. Dell'Era and R. Verganti, "Collaborative Strategies in Design-intensive Industries: Knowledge Diversity and Innovation," Long Range Plann., vol. 43, no. 1, pp. 123-141, 2010.

11.B. Andres and R. Poler, "Improving the collaborative network performance through the activation of compatible strategies," Int. J. Eng. Manag. Econ., vol. 5, no. 1/2, pp. 35-47, 2015.

12.B. Andres, R. Poler, and R. Sanchis, "Collaborative Strategies Alignment to enhance the Collaborative Network agility and resilience," IFIP Adv. Inf. Commun. Technol., vol. 463, pp. 88-99, 2015.

13.B. Andres and R. Poler, "Modelling the Strategies Alignment process in the Collaborative Network context," in ICIEOM-CIO-IIIE International Conference. Engineering Systems and Networks: The way ahead for industrial engineering and operations management, 2015.

14.J. W. Forrester, Industrial dynamics. Cambridge, MA: MIT press., 1961.

15.AnyLogic, “AnyLogic.” 2015.

16.Lazarus Free Pascal, "Lazarus." 2015. 